\title{
Noncancellation of infrared singularities in collisions of massive quarks
}

\author{
Fabrizio Caola $\odot,{ }^{1,2}$ Kirill Melnikov, ${ }^{3}$ Davide Napoletano, ${ }^{4}$ and Lorenzo Tancredi ${ }^{1}$ \\ ${ }^{1}$ Rudolf Peierls Centre for Theoretical Physics, Clarendon Laboratory, \\ Parks Road, Oxford OX1 3PU, United Kingdom \\ ${ }^{2}$ Wadham College, University of Oxford, Oxford OX1 3PN, United Kingdom \\ ${ }^{3}$ Institute for Theoretical Particle Physics, KIT, 76128 Karlsruhe, Germany \\ ${ }^{4}$ Università Milano-Bicocca \& INFN, Piazza della Scienza 3, Milano 20126, Italia
}

(Received 30 November 2020; accepted 9 February 2021; published 11 March 2021)

\begin{abstract}
We discuss the infrared structure of processes with massive quarks in the initial state. It is well known that, starting from next-to-next-to-leading order in perturbative QCD, such processes exhibit a violation of the Bloch-Nordsieck theorem, in that the sum of real and virtual contributions to partonic cross sections contains uncanceled infrared singularities. The main purpose of this paper is to present a simple physical argument that elucidates the origin of these singularities and simplifies the derivation of infrared-singular contributions to heavy-quark initiated cross sections.
\end{abstract}

DOI: 10.1103/PhysRevD.103.054013

\section{INTRODUCTION AND GENERAL CONSIDERATIONS}

The infrared structure of perturbative gauge theories is a fascinating topic which received significant attention since the early days of QCD [1,2]. In the 70s, the observation of factorization of soft and collinear divergences in deepinelastic scattering $[3,4]$ paved the way for a new understanding of the perturbative structure of gauge theories, leading to the promotion of the naive parton model [5] to a well-defined approximation rooted in a fully consistent quantum theory of strong interactions.

Generalization of these results to the more complicated case of hadron-hadron collisions [6-9] resulted in a better understanding on the universal pattern of factorization and cancellation of long-distance effects in perturbative QCD calculations. This understanding was eventually distilled into "theorems" [10-13] that state that (potential) logarithmic sensitivity to long-distance effects is absent in sufficiently inclusive observables in hard scattering processes. This remarkable fact is the foundation of modern collider phenomenology as it allows us to provide firstprinciples improvements of the theoretical description of hadron collisions by refining predictions for partonic scattering cross sections in QCD perturbation theory.

Given the prominence of these theorems in modern collider physics, it is useful to inquire about their

Published by the American Physical Society under the terms of the Creative Commons Attribution 4.0 International license. Further distribution of this work must maintain attribution to the author(s) and the published article's title, journal citation, and DOI. Funded by SCOAP ${ }^{3}$. limitations. Such a question, albeit being interesting in its own right, may also have practical consequences for the precision physics program at current and future colliders by, e.g., informing us about ultimate limits in precision that improvements in perturbative computations alone can possibly provide.

Indeed, while the aforementioned theorems are very solid in the case of lepton-lepton or lepton-hadron collisions, the situation is more delicate in case of hadron-hadron collisions; see, e.g., [11,13]. In fact, it was argued that, at sufficiently high orders in perturbation theory, combining real and virtual corrections within the framework of collinear factorization may be insufficient to get rid of the infrared sensitivity, even for inclusive observables [14-16].

For processes involving massless partons in the initial state, our current understanding of the soft-collinear structure of QCD implies that these issues can only appear at third or higher orders in QCD perturbation theory. ${ }^{1}$ However, the situation is very different if one considers massive quarks in the initial state. In this case, it was pointed out long ago that starting from second order in QCD perturbation theory the sum of real and virtual corrections is not free of infrared singularities. As a consequence, "standard" perturbative calculations in this case become insufficient beyond next-to-leading order, even for the simplest partonic processes [19].

This problem received a lot of attention in the past [20], and several formal ways of dealing with it have been proposed [21]. The goal of this paper is to present a derivation of the divergent contribution to the cross section of a process with

\footnotetext{
${ }^{1}$ At third order, they are only relevant for processes involving a nontrivial color structure [16-18].
} 
two heavy quarks in the initial state that, in our opinion, is remarkably simple and physically transparent.

Our argument is inspired by recent work on infrared subtraction schemes for higher order calculations [22,23] and, in a nutshell, consists in connecting infrared singular contributions of a process where infrared finiteness is guaranteed to infrared singular contributions of a process initiated by the collision of two massive quarks. In what follows, we focus on the Drell-Yan process where a virtual photon is produced in the collision of a quark and an antiquark. The simplicity of this process allows us to present our argument with a minimal amount of technical overhead.

The remainder of this paper is organized as follows. In Sec. II A, we show by an explicit computation that there are no uncanceled infrared singularities at next-to-leading order (NLO) QCD for the Drell-Yan process with massive initial-state quarks and comment on the generalization of this result to arbitrary processes. We also argue that the absence of infrared singularities in the production process $q \bar{q} \rightarrow V+X$ at NLO QCD can be naturally understood if the absence of infrared singularities in the decay process $V \rightarrow q \bar{q}+X$ is taken for granted. In Sec. II B, we generalize this argument to the next-to-next-to-leading order (NNLO) case and show that at NNLO there is only one potential source of noncanceling soft singularities. In Sec. III, we explicitly compute the infrared singular contribution to the Drell-Yan cross section and comment on the result. We conclude in Sec. IV. The analytic continuation of the one-loop integrals required for our analysis is discussed in the Appendix.

\section{DRELL-YAN PROCESS WITH INITIAL-STATE MASSIVE QUARKS}

We begin with the discussion of the infrared structure of the process

$$
q\left(p_{1}\right)+\bar{q}\left(p_{2}\right) \rightarrow V\left(p_{V}\right)+X,
$$

where $q, \bar{q}$ are massive quarks with $p_{1}^{2}=p_{2}^{2}=m_{q}^{2}$ and $V$ is a virtual photon ${ }^{2}$ with $p_{V}^{2}=m_{V}^{2}$. Since there are no massless partons in the initial state of this process, no collinear renormalization of parton distribution functions is required. The perturbative expansion of the partonic cross section for this process reads

$$
\mathrm{d} \sigma=\mathrm{d} \sigma_{\mathrm{LO}}+\mathrm{d} \sigma_{\mathrm{NLO}}+\mathrm{d} \sigma_{\mathrm{NNLO}}+\mathcal{O}\left(\alpha_{s}^{3}\right) .
$$

\section{A. Next-to-leading order}

We start by considering NLO QCD contributions to the cross section of the process in Eq. (2.1). We write them as

\footnotetext{
${ }^{2}$ Our argument applies verbatim for any (massive) colorsinglet final state $V$.
}

$$
\mathrm{d} \sigma_{\mathrm{NLO}}=\mathrm{d} \sigma_{\mathrm{V}}+\mathrm{d} \sigma_{\mathrm{R}}
$$

The first term on the rhs of Eq. (2.3) represents UVrenormalized contributions of one-loop virtual corrections. It reads [24]

$\mathrm{d} \sigma_{\mathrm{V}}=\frac{\alpha_{s}(\mu)}{2 \pi}\left\{-\frac{2 C_{F}}{\epsilon}\left[\frac{1}{2 v} \ln \left(\frac{1-v}{1+v}\right)+1\right]\right\} \mathrm{d} \sigma_{\mathrm{LO}}+\mathrm{d} \sigma_{V, \text { fin }}$,

where $\epsilon=(4-d) / 2$ and $d$ is the dimensionality of spacetime. Also, $C_{F}=4 / 3$ is the Casimir invariant of the $S U(3)$ gauge group of QCD, $v=\sqrt{1-m^{4} /\left(p_{1} \cdot p_{2}\right)^{2}}$ and $\mathrm{d} \sigma_{V, \text { fin }}$ is finite in the $\epsilon \rightarrow 0$ limit. The $1 / \epsilon$ pole in Eq. (2.4) is of infrared origin; it is well known that it is canceled by a similar divergence in the real emission contribution $\mathrm{d} \sigma_{\mathrm{R}}$.

To illustrate this, consider the real emission process $^{3}$

$$
q\left(p_{1}\right)+\bar{q}\left(p_{2}\right) \rightarrow V\left(p_{V}\right)+g\left(p_{g}\right)
$$

and write

$$
\begin{aligned}
\mathrm{d} \sigma_{\mathrm{R}}= & \frac{1}{4 J} \int\left[\mathrm{d} p_{V}\right]\left[\mathrm{d} p_{g}\right] \bar{\sum}\left|\mathcal{M}_{0}\left(p_{1}, p_{2} ; p_{V}, p_{g}\right)\right|^{2} \\
& \times(2 \pi)^{d} \delta_{d}\left(p_{1}+p_{2}-p_{V}-p_{g}\right),
\end{aligned}
$$

where $J=p_{1} \cdot p_{2} v$ is the flux factor, $\left[\mathrm{d} p_{V, g}\right]=$ $\mathrm{d}^{d-1} p_{V, g} /\left((2 \pi)^{d-1} 2 E_{V, g}\right)$ are the phase-space elements of the virtual photon and the gluon, respectively, $\bar{\Sigma}$ indicates the sum (average) over final-state (initial-state) colors and polarizations, and $\mathcal{M}_{0}$ is the tree-level scattering amplitude for the process Eq. (2.5). When the emitted gluon becomes soft, $E_{g} \rightarrow 0$, the matrix element $\left|\mathcal{M}_{0}\right|^{2}$ scales as $E_{g}^{-2}$, and Eq. (2.6) develops a logarithmic singularity. To expose it, we work in the partonic center-of-mass frame, separate the integration over the gluon energy, and write

$$
\mathrm{d} \sigma_{\mathrm{R}}=\int \frac{\mathrm{d} E_{g}}{E_{g}^{1+2 \epsilon}} \frac{\mathrm{d} \Omega_{g}^{(d-1)}}{2(2 \pi)^{d-1}} F_{g}^{(d)}\left(p_{1}, p_{2}, p_{V} ; p_{g}\right),
$$

where

$$
\begin{aligned}
F_{g}^{(d)}\left(p_{1}, p_{2}, p_{V} ; p_{g}\right)= & \frac{1}{4 J}\left[\mathrm{~d} p_{V}\right] E_{g}^{2} \bar{\sum}\left|\mathcal{M}_{0}\left(p_{1}, p_{2} ; p_{V}, p_{g}\right)\right|^{2} \\
& \times(2 \pi)^{d} \delta_{d}\left(p_{1}+p_{2}-p_{V}-p_{g}\right) .
\end{aligned}
$$

To extract infrared divergences from Eq. (2.7), we write

$\mathrm{d} \sigma_{\mathrm{R}}=\int_{0}^{E_{\max }} \frac{\mathrm{d} E_{g}}{E_{g}^{1+2 \epsilon}} \frac{\mathrm{d} \Omega_{g}^{(3)}}{16 \pi^{3}} \lim _{E_{g} \rightarrow 0}\left[F_{g}^{(4)}\left(p_{1}, p_{2}, p_{V} ; p_{g}\right)\right]+\mathrm{d} \sigma_{\mathrm{R}}^{\text {fin }}$,

\footnotetext{
${ }^{3}$ We only consider the corrections to the $q \bar{q}$ channel, since the $q g$ channel is infrared finite.
} 
where the second contribution is finite and the first one is divergent. We rewrite it as

$$
\int_{0}^{E_{\max }} \frac{\mathrm{d} E_{g}}{E_{g}^{1+2 \epsilon}} \frac{\mathrm{d} \Omega_{g}^{(3)}}{16 \pi^{3}} \lim _{E_{g} \rightarrow 0}\left[F_{g}^{(4)}\left(p_{1}, p_{2}, p_{V} ; p_{g}\right)\right]=\mathrm{d} \sigma_{R}^{\mathrm{div}}+\cdots,
$$

where

$$
\mathrm{d} \sigma_{\mathrm{R}}^{\mathrm{div}}=-\frac{1}{2 \epsilon} \int \frac{\mathrm{d} \Omega_{g}^{(3)}}{16 \pi^{3}} \lim _{E_{g} \rightarrow 0}\left[F_{g}^{(4)}\left(p_{1}, p_{2}, p_{V} ; p_{g}\right)\right],
$$

and the ellipses in Eq. (2.10) stand for finite terms.

To proceed further, we recall that in the soft limit scattering amplitudes obey the well-known factorization formula

$$
\begin{aligned}
& \mathcal{M}_{0}\left(p_{1}, p_{2} ; p_{V}, p_{g^{a}}\right) \\
& \quad \approx g_{s}^{2} \varepsilon^{\mu} J_{\mu}^{a,(0)}\left(p_{1}, p_{2} ; p_{g}\right) \mathcal{M}_{0}\left(p_{1}, p_{2} ; p_{V}\right),
\end{aligned}
$$

where $\varepsilon^{\mu}$ is the gluon polarization vector and $a$ is its color index. The tree-level soft current reads

$$
J_{\mu}^{a,(0)}\left(p_{1}, p_{2} ; p_{g}\right)=\sum_{i=1}^{2} T_{i}^{a} \frac{p_{i, \mu}}{p_{i} \cdot p_{g}}
$$

where $T_{i}^{a}$ is the color charge of particle $i$. In our case, $T_{1}^{a}=t_{21}^{a}$ and $T_{2}^{a}=-t_{12}^{a}$, where $t_{i j}^{a}$ is the matrix element of an $S U(3)$ algebra generator in the fundamental representation. ${ }^{4}$ This immediately allows us to rewrite Eq. (2.11) as

$$
\mathrm{d} \sigma_{\mathrm{R}}^{\mathrm{div}}=\operatorname{Eik}_{0}\left(p_{1}, p_{2}\right) \times \mathrm{d} \sigma_{\mathrm{LO}},
$$

where

$$
\begin{aligned}
\operatorname{Eik}_{0}\left(p_{1}, p_{2}\right)= & -\frac{\alpha_{s}(\mu)}{2 \pi} \frac{C_{F}}{\epsilon} \int \frac{\mathrm{d} \Omega_{3, g}}{4 \pi} E_{g}^{2}\left[\frac{2\left(p_{1} \cdot p_{2}\right)}{\left(p_{1} \cdot p_{g}\right)\left(p_{2} \cdot p_{g}\right)}\right. \\
& \left.-\frac{m_{q}^{2}}{\left(p_{1} \cdot p_{g}\right)^{2}}-\frac{m_{q}^{2}}{\left(p_{2} \cdot p_{g}\right)^{2}}\right] .
\end{aligned}
$$

We parametrize momenta in Born kinematics as $p_{1,2}=m_{V} / 2(1,0,0, \pm \beta)$, with $\beta=\sqrt{1-4 m_{q}^{2} / m_{V}^{2}}$ and $p_{g}=E_{g}(1, \sin \theta, 0, \cos \theta)$. A straightforward integration over the gluon emission angle leads to

\footnotetext{
${ }^{4}$ For more details on the color notation, see, e.g., [25].
}

$\mathrm{d} \sigma_{\mathrm{R}}^{\mathrm{div}}=\frac{\alpha_{s}(\mu)}{2 \pi} \times \frac{2 C_{F}}{\epsilon}\left[\frac{1+\beta^{2}}{2 \beta} \ln \left(\frac{1-\beta}{1+\beta}\right)+1\right] \mathrm{d} \sigma_{\mathrm{LO}}$.

The cancellation of soft singularities in the NLO cross section can be observed upon combining $\mathrm{d} \sigma_{\mathrm{V}}$ from Eq. (2.4) and $\mathrm{d} \sigma_{\mathrm{R}}^{\text {div }}$ from Eq. (2.16) and using the relation between $v$ and $\beta, v=2 \beta /\left(1+\beta^{2}\right)$, which implies

$$
\frac{1+\beta^{2}}{2 \beta} \ln \left(\frac{1-\beta}{1+\beta}\right)=\frac{1}{2 v} \ln \left(\frac{1-v}{1+v}\right) .
$$

We also note that the cancellation of infrared divergences occurs in a much broader context than what we discuss here for the Drell-Yan process. Indeed, by considering a generalization of Eq. (2.4) to $2 \rightarrow n$ processes as described in Ref. [24], and adapting Eq. (2.13) to this case, it is straightforward to prove the cancellation of infrared divergences for arbitrary processes with massive quarks in the initial state.

We will now reanalyze the NLO case from a perspective that will be helpful for deriving the infrared divergent contribution to the NNLO cross section. To this end, instead of considering the production process $q \bar{q} \rightarrow V+X$, we start with its decay counterpart $V\left(p_{V}\right) \rightarrow q\left(p_{1}\right)+\bar{q}\left(p_{2}\right)+X$. We use the optical theorem and obtain the total decay rate of the above process from the imaginary part of the timeordered correlator of two vector currents. Since such correlator cannot have infrared divergences, we conclude that the decay rate is free of infrared singularities as well. Writing the decay rate as the sum of virtual and realemission contributions, we conclude that $\mathrm{d} \sigma_{\mathrm{V}}^{\text {decay }}+\mathrm{d} \sigma_{\mathrm{R}}^{\text {decay }}$ is infrared finite.

We now want to relate $\mathrm{d} \sigma_{\mathrm{V}}^{\text {decay }}, \mathrm{d} \sigma_{\mathrm{R}}^{\text {decay }}$ to their counterparts in the production case Eqs. (2.4) and (2.6). For virtual corrections, this relation is obvious. Indeed, one-loop corrections to the $\gamma^{*} \rightarrow q \bar{q}$ vertex are described by a single form factor $F_{V}$ that only depends on the invariant mass of the virtual photon $m_{V}^{2}$. ${ }^{5}$ Hence, this form factor is identical for the production $\left(q \bar{q} \rightarrow \gamma^{*}\right)$ and decay $\left(\gamma^{*} \rightarrow q \bar{q}\right)$ processes. We conclude that the infrared structure of the decay rate $\mathrm{d} \sigma_{\mathrm{V}}^{\text {decay }}$ and the production cross section $\mathrm{d} \sigma_{\mathrm{V}}$ is the same. Therefore,

$$
\begin{aligned}
\mathrm{d} \sigma_{\mathrm{V}}^{\text {decay }} & =F_{V}\left(m_{V}^{2}, \epsilon\right) \mathrm{d} \sigma_{\mathrm{LO}}^{\text {decay }}+\cdots \quad \text { and } \\
\mathrm{d} \sigma_{\mathrm{V}} & =F_{V}\left(m_{V}^{2}, \epsilon\right) \mathrm{d} \sigma_{\mathrm{LO}}+\cdots,
\end{aligned}
$$

where the ellipses stand for finite contributions.

To make use of the finite nature of NLO corrections to the decay as an explanation of why NLO corrections to the

\footnotetext{
${ }^{5}$ The dependence of the form factor on quark masses is not relevant for this discussion.
} 
production are finite, we need to understand how the real emission contribution to the decay rate changes when we move heavy quarks into the initial state and the vector boson into the final state which is required for calculating the production cross section. Since we are only interested in the divergent contribution to the cross section, we require this crossing in the soft limit. We note that the tree-level soft current Eq. (2.13) is homogeneous in the hard momenta $p_{1,2}$ (and it does not depend on the momenta of the color singlet), so it does not change under the replacement $p_{i} \rightarrow-p_{i}$. Moreover, the phase space of the Born process decouples from the eikonal factor and the gluon phase space in the soft limit. It follows that

$$
\begin{aligned}
\mathrm{d} \sigma_{\mathrm{R}}^{\text {decay }} & =\operatorname{Eik}_{0}\left(p_{1}, p_{2}\right) \times \mathrm{d} \sigma_{\mathrm{LO}}^{\text {decay }}+\cdots \quad \text { and } \\
\mathrm{d} \sigma_{\mathrm{R}} & =\operatorname{Eik}_{0}\left(p_{1}, p_{2}\right) \times \mathrm{d} \sigma_{\mathrm{LO}}+\cdots,
\end{aligned}
$$

where ellipses stand for finite contributions and the function $\mathrm{Eik}_{0}$ is defined in Eq. (2.15). Since $\mathrm{d} \sigma_{\mathrm{V}}^{\text {decay }}+\mathrm{d} \sigma_{\mathrm{R}}^{\text {decay }}$ is free of infrared divergences, it follows from Eqs (2.18) and (2.19) that

$$
F_{V}\left(m_{V}^{2}, \epsilon\right)+\operatorname{Eik}_{0}\left(p_{1}, p_{2}\right)
$$

is infrared finite. Without any additional computation, this ensures that the $\mathcal{O}\left(\alpha_{s}\right)$ contributions to the cross section of $q \bar{q} \rightarrow V+X$ with massive initial state quarks are finite as well. In the next section, we generalize this analysis to nextto-next-to-leading order.

\section{B. Next-to-next-to-leading order contributions to the production cross section}

Consider the NNLO QCD contributions to the cross section of the production process $q \bar{q} \rightarrow V+X$. In full analogy to the NLO case discussed in the previous section, we split $\mathrm{d} \sigma_{\mathrm{NNLO}}$ into double-virtual, double-real, and realvirtual contributions,

$$
\mathrm{d} \sigma_{\mathrm{NNLO}}=\mathrm{d} \sigma_{\mathrm{VV}}+\mathrm{d} \sigma_{\mathrm{RR}}+\mathrm{d} \sigma_{\mathrm{RV}} .
$$

In this equation, the double-virtual term $\mathrm{d} \sigma_{\mathrm{VV}}$ is proportional to the two-loop form factor for the $q \bar{q} \rightarrow V$ process. The double-real term $\mathrm{d} \sigma_{\mathrm{RR}}$ is proportional to the tree-level matrix element for the process

$$
q\left(p_{1}\right)+\bar{q}\left(p_{2}\right) \rightarrow V\left(p_{V}\right)+f_{i}\left(p_{i}\right)+f_{j}\left(p_{j}\right),
$$

where $\left(f_{j}, f_{j}\right) \in\left\{(g, g),\left(q_{i}, \bar{q}_{j}\right)\right\}$ and $q_{i}$ is a generic (massive or massless) quark. Finally, the real-virtual contribution $\mathrm{d} \sigma_{\mathrm{RV}}$ is proportional to the one-loop matrix element for the process

$$
q\left(p_{1}\right)+\bar{q}\left(p_{2}\right) \rightarrow V\left(p_{V}\right)+g\left(p_{g}\right) .
$$

In principle, one can study the infrared structure of the various contributions at this perturbative order by extending the NLO analysis presented at the beginning of the previous section to one order higher. However, it is much easier and more transparent to reuse the connection between the production and decay processes as was done at the end of the previous section. For this reason, we consider the NNLO QCD contributions to the decay process $V \rightarrow q \bar{q}+X$, which is finite, and write

$$
\mathrm{d} \sigma_{\mathrm{NNLO}}^{\text {decay }}=\mathrm{d} \sigma_{\mathrm{VV}}^{\text {decay }}+\mathrm{d} \sigma_{\mathrm{RR}}^{\text {decay }}+\mathrm{d} \sigma_{\mathrm{RV}}^{\text {decay }} .
$$

We then compare each contribution to its counterpart in the production case. The results of this comparison can be summarized as follows:

(a) All infrared singularities of the double-virtual contributions come from the one- and two-loop $V q \bar{q}$ form factors. Since the form factor is the same for the $V \rightarrow$ $q+\bar{q}$ and $q+\bar{q} \rightarrow V$ processes, the infrared structure of $\mathrm{d} \sigma_{\mathrm{VV}}^{\text {decay }}$ and $\mathrm{d} \sigma_{\mathrm{VV}}$ is identical.

(b) In the double-real contribution, infrared singularities appear when either one or two final state gluons become soft, or when a massless final state quark pair becomes soft. The case of one-gluon emission is described by the tree-level current Eq. (2.13). The emission of two soft partons is described by a doublesoft current [26] that is homogeneous in the momenta of the external hard partons. Similar to the NLO case described above, this implies that the infrared structure of $\mathrm{d} \sigma_{\mathrm{RR}}^{\text {decay }}$ and $\mathrm{d} \sigma_{\mathrm{RR}}$ is identical.

(c) The real-virtual contribution contains both explicit $1 / \epsilon$ infrared poles in the $q \bar{q} \rightarrow V+g$ one-loop amplitude and implicit singularities that only appear after integrating over the soft region of the gluon phase space. As long as the gluon is hard, this integration does not introduce any divergence and only explicit singularities are relevant. These singularities cancel against single soft-gluon emission in the double-real contribution along the lines of the NLO case described in the previous section. As we explained there, this cancellation occurs for both the production and the decay processes.

The only contribution that we still need to discuss is a one-loop correction to the emission of a soft gluon. In this case, we cannot invoke the crossing argument to conclude that the production and decay processes share the same infrared structure because the analytic structure of loop amplitudes is nontrivial and care is needed to relate the production and decay cases.

Hence, we conclude that the infrared structure of the production and decay processes is identical, except for possible contributions that originate from crossing the $V \rightarrow q+\bar{q}+g$ one-loop amplitude into the $q+\bar{q} \rightarrow$ $V+g$ one, in the kinematic configuration where $g$ is soft. Since the total rate for $V \rightarrow q+\bar{q}+X$ is finite, this implies that the only potential noncanceling infrared singularities in $q+\bar{q} \rightarrow V+X$ at NNLO must be related to this crossing. Below we show that the analytic continuation from decay to production kinematics is indeed nontrivial, and that it 

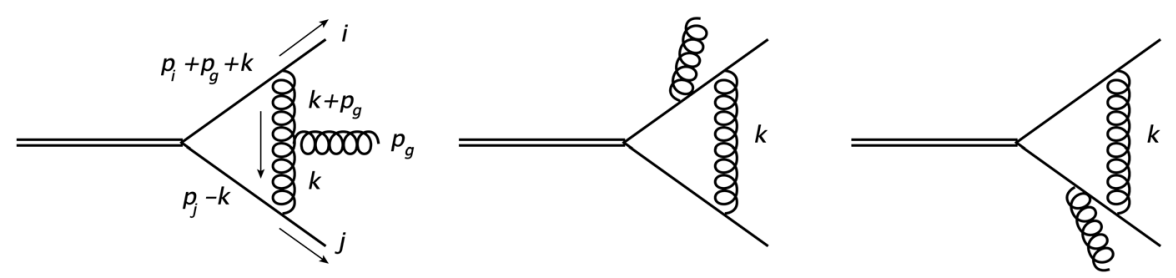

FIG. 1. Diagrams contributing to the one-loop soft current. $i$ and $j$ are hard eikonal lines, $p_{g}$ and $k$ are soft; see text for details.

leads to an uncanceled $1 / \epsilon$ infrared singularity in the production cross section. ${ }^{6}$

\section{THE ONE-LOOP SOFT CURRENT AND ITS CROSSING}

In this section, we study one-loop corrections to soft-gluon emission. More precisely, following the discussion in the previous section, we investigate whether additional infrared divergences can be generated by crossing the one-loop decay amplitude $\mathcal{M}_{1}\left(p_{V} ; p_{1}, p_{2}, p_{g}\right)$ into the amplitude $\mathcal{M}_{1}\left(p_{1}, p_{2} ; p_{V}, p_{g}\right)$ that describes the production process.

Similar to the tree-level case Eq. (2.12), the one-loop amplitude $\mathcal{M}_{1}$ also factorizes in the soft limit ${ }^{7}$

$$
\begin{aligned}
\mathcal{M}_{1}\left(p_{V} ; p_{1}, p_{2}, p_{g}\right) \approx & g_{s}^{2} \varepsilon^{\mu}\left[J_{\mu}^{a,(0)}\left(p_{1}, p_{2} ; p_{g}\right) \mathcal{M}_{1}\left(p_{V} ; p_{1}, p_{2}\right)\right. \\
& \left.+g_{s}^{2} J_{\mu}^{a,(1)}\left(p_{1}, p_{2} ; p_{g}\right) \mathcal{M}_{0}\left(p_{V} ; p_{1}, p_{2}\right)\right]
\end{aligned}
$$

We stress that $\mathcal{M}_{1}$ in the above equation is the scattering amplitude of the decay process and we intend to get the production amplitude by crossing.

The tree-level current $J_{\mu}^{a,(0)}$ is given in Eq. (2.13); as discussed in Secs. II A, II B, it leads to the same infrared divergences in the production and decay cases. Hence, we only need to focus on the second term on the right-hand side of Eq. (3.1) that describes the one-loop correction to the soft current.

To compute the one-loop soft current $J_{\mu}^{a,(1)}$, one needs to consider the non-Abelian part of the diagrams shown in Fig. 1, in the limit where both virtual and real gluons are soft [27]. The result reads

$$
\begin{aligned}
& J^{a,(1), \mu}\left(p_{1}, p_{2} ; p_{g}\right) \\
& \quad=i f_{a b c} \sum_{\substack{i, j=1 \\
i \neq j}}^{2} T_{i}^{b} T_{j}^{c}\left(\frac{p_{i}^{\mu}}{p_{i} \cdot p_{g}}-\frac{p_{j}^{\mu}}{p_{j} \cdot p_{g}}\right) g_{i j}^{(1)}\left(\epsilon, p_{g} ; p_{i}, p_{j}\right) \\
& \quad=g_{12}^{(1)}\left(\epsilon, p_{g} ; p_{1}, p_{2}\right) C_{A} J^{a,(0), \mu}\left(p_{1}, p_{2} ; p_{g}\right)
\end{aligned}
$$

\footnotetext{
${ }^{6}$ We note that similar arguments suggest that other partonic channels, i.e., $q g$ and $g g$, are infrared finite.

${ }^{7}$ In this equation, $g_{s}$ is the bare strong coupling. Since we are interested in infrared effects, we do not discuss renormalization.
}

where $f_{a b c}$ are the $S U(3)$ structure constants and $g_{i j}^{(1)}$ is a function that will be specified later. We stress that $J_{\mu}^{a,(1)}$ is purely non-Abelian. This feature is expected because in an Abelian theory the tree-level soft current does not receive corrections. Since, as we argued at the beginning of this section, Eq. (3.2) provides the only source of noncanceling soft singularities for the process $q \bar{q} \rightarrow V+X$ with massive initial particles, we recover the classic result that in the Abelian (e.g., QED) case the NNLO cross section for the collision of two massive partons is infrared finite.

We continue with the non-Abelian case. Following the argument of Sec. II B, we investigate whether Eq. (3.2) leads to the same infrared structure for the decay and production processes. Since $J^{a,(0), \mu}$ is invariant under $p_{1,2} \rightarrow-p_{1,2}$, any potential difference must come from the crossing of $g_{12}^{(1)}$. It is easy to see that, at NNLO, only the real part of $g_{12}^{(1)}$ contributes to the cross section; for this reason, we investigate the behavior of $\Re\left[g_{12}^{(1)}\right]$ under $p_{1,2} \rightarrow-p_{1,2}$ transformation.

It is instructive to consider first the case of massless quarks. For $m_{q}=0$, the function $g_{12}^{(1)}$ reads [27]

$$
\begin{aligned}
g_{12}^{(1)}\left(\epsilon, p_{g} ; p_{1}, p_{2}\right)= & -\frac{1}{16 \pi^{2}} \frac{1}{\epsilon^{2}} \frac{\Gamma^{3}(1-\epsilon) \Gamma^{2}(1+\epsilon)}{\Gamma(1-2 \epsilon)} \\
& \times\left[\frac{\left(-s_{12}-i \delta\right)}{\left(-s_{1 g}-i \delta\right)\left(-s_{2 g}-i \delta\right)}\right]^{\epsilon},
\end{aligned}
$$

with $s_{i j}=2 p_{i} \cdot p_{j}$. This implies

$\Re\left[g_{12}^{(1)}\left(\epsilon, p_{g} ;-p_{1},-p_{2}\right)\right]=\Re\left[g_{12}^{(1)}\left(\epsilon, p_{g} ; p_{1}, p_{2}\right)\right]$.

The argument of Sec. II B then allows us to reproduce the standard result that for massless quarks the cross section for the process $q+\bar{q} \rightarrow V$ is free from soft singularities at NNLO. ${ }^{8}$

We continue with the case $m_{q} \neq 0$. In this case, we follow Ref. [28] and write $g_{12}^{(1)}$ as

\footnotetext{
${ }^{8}$ To remove initial-state collinear singularities, one still needs to redefine parton distribution functions in the case of massless particles collisions.
} 


$$
g_{12}^{(1)}\left(\epsilon, p_{g} ; p_{1}, p_{2}\right)=\sum_{i=1}^{3} f_{i}\left(p_{g} ; p_{1}, p_{2}\right) M_{i}\left(\epsilon, p_{g} ; p_{1}, p_{2}\right),
$$

where $M_{i}$ are defined as

$$
\begin{aligned}
& M_{1}\left(\epsilon, p_{g} ; p_{1}, p_{2}\right)=\int \frac{d^{d} k}{(2 \pi)^{d}} \frac{1}{\left[k^{2}+i \delta\right]\left[\left(k+p_{g}\right)^{2}+i \delta\right]\left[-2 p_{2} \cdot k+i \delta\right]}, \\
& M_{2}\left(\epsilon, p_{g} ; p_{1}, p_{2}\right)=\int \frac{d^{d} k}{(2 \pi)^{d}} \frac{1}{\left[k^{2}+i \delta\right]\left[2 p_{1} \cdot k+2 p_{1} \cdot p_{g}+i \delta\right]\left[-2 p_{2} \cdot k+i \delta\right]}, \\
& M_{3}\left(\epsilon, p_{g} ; p_{1}, p_{2}\right)=\int \frac{d^{d} k}{(2 \pi)^{d}} \frac{1}{\left[k^{2}+i \delta\right]\left[\left(k+p_{g}\right)^{2}+i \delta\right]\left[2 p_{1} \cdot k+2 p_{2} \cdot p_{g}+i \delta\right]\left[-2 p_{2} \cdot k+i \delta\right]},
\end{aligned}
$$

and $f_{i}$ are rational functions of $p_{i} \cdot p_{j}, p_{i} \cdot p_{g}$. Since $g_{12}^{(1)}$ has to be computed using eikonal vertices [27], it follows that

$$
\begin{array}{r}
f_{1}\left(p_{g} ;-p_{1},-p_{2}\right)=-f_{1}\left(p_{g} ; p_{1}, p_{2}\right), \\
f_{2,3}\left(p_{g} ;-p_{1},-p_{2}\right)=f_{2,3}\left(p_{g} ; p_{1}, p_{2}\right) .
\end{array}
$$

The explicit form of $f_{i}$ can be found in Ref. [28], but it is not needed for our argument.

Using Eqs. (3.5)-(3.7), one can show by analytic continuation of the $M_{j}$ integrals that the function $g_{12}^{(1)}$ changes in the following way:

$$
g_{12}^{(1)}\left(\epsilon, p_{g} ;-p_{1},-p_{2}\right)=e^{-2 i \epsilon \pi} g_{12}^{(1)}\left(\epsilon, p_{g} ; p_{1}, p_{2}\right) .
$$

This is worked out explicitly in the Appendix. To proceed further, we write the (decay) function $g_{12}^{(1)}$ as

$$
g_{12}^{(1)}\left(\epsilon, p_{g} ; p_{1}, p_{2}\right)=\frac{\alpha_{s}}{2 \pi} E_{g}^{-2 \epsilon} \sum_{k=-2}^{\infty}\left[\mathfrak{r}_{k}+i \cdot \dot{\mathfrak{t}}_{k}\right] \epsilon^{k},
$$

with $\mathfrak{r}$ and $\mathfrak{i}$ real and $\mathfrak{i}_{-2}=0$; see the Appendix. Using Eqs. (3.8) and (3.9), we can then write the difference between the real parts of the functions $g_{12}$ required to describe the production and the decay processes as

$$
\begin{array}{r}
\Re\left[g_{12}^{(1)}\left(\epsilon, p_{g} ;-p_{1},-p_{2}\right)\right]-\mathfrak{R}\left[g_{12}^{(1)}\left(\epsilon, p_{g} ; p_{1}, p_{2}\right)\right] \\
=\frac{\alpha_{s}}{2 \pi}\left|\frac{s_{12}}{s_{1 g} s_{2 g}}\right|^{\epsilon}\left[-2 \pi^{2} \cdot \mathfrak{r}_{-2}+2 \pi \cdot \mathfrak{i}_{-1}+\mathcal{O}(\epsilon)\right] .
\end{array}
$$

Since the real part of $g_{12}^{(1)}$ at order $\mathcal{O}\left(\epsilon^{0}\right)$ contributes to divergences of the cross section or decay rate at order $1 / \epsilon$, the argument presented in Sec. II B implies that the second line of Eq. (3.10) gives rise to a noncanceling infrared divergence in the NNLO cross section for the $q+\bar{q} \rightarrow V$ process with massive quarks in the initial state.
This noncanceled singularity is controlled by the coefficients $\mathfrak{x}_{-2}$ and $\mathfrak{i}_{-1}$. They can be immediately obtained by matching Eq. (3.1) to the universal expression for the infrared poles of one-loop amplitudes [24]. We obtain

$$
\mathfrak{r}_{-2}=-\frac{1}{2}, \quad \mathfrak{i}_{-1}=\pi\left(\frac{1}{2 v}-1\right),
$$

with $v$ defined immediately after Eq. (2.4). We work in the center-of-mass frame of the two quarks and rewrite Eq. (3.10) as

$$
\begin{aligned}
\Re & {\left[g_{12}^{(1)}\left(\epsilon, p_{g} ;-p_{1},-p_{2}\right)\right] } \\
& =\Re\left[g_{12}^{(1)}\left(\epsilon, p_{g} ; p_{1}, p_{2}\right)\right]+\frac{\alpha_{s}}{2 \pi} E_{g}^{-2 \epsilon}\left[\left(\frac{1-v}{v}\right) \pi^{2}+\mathcal{O}(\epsilon)\right] .
\end{aligned}
$$

To find the contribution of the last term in Eq. (3.12) to the cross section, we note that the soft current at one loop is proportional to the tree-level one, cf. Eq. (3.2). As a consequence, we can read off the required result directly from Eq. (2.16) that describes the NLO calculation. ${ }^{9}$ Therefore, we write the real-virtual contribution to the decay process as

$$
\mathrm{d} \sigma_{\mathrm{RV}}^{\text {decay }}=\operatorname{Eik}_{1}\left(p_{1}, p_{2}\right) \times \mathrm{d} \sigma_{\mathrm{LO}}^{\text {decay }}+\cdots,
$$

where the ellipses stand for finite contributions. The real-virtual contribution to the production process is given by

$$
\begin{aligned}
\mathrm{d} \sigma_{\mathrm{RV}} & =\operatorname{Eik}_{1}\left(-p_{1},-p_{2}\right) \times \mathrm{d} \sigma_{\mathrm{LO}} \\
& =\operatorname{Eik}_{1}\left(p_{1}, p_{2}\right) \times \mathrm{d} \sigma_{\mathrm{LO}}+\Delta\left[\mathrm{d} \sigma_{\mathrm{RV}}^{\mathrm{div}}\right]+\cdots
\end{aligned}
$$

\footnotetext{
${ }^{9}$ Note that the additional $E_{g}^{-2 \epsilon}$ factor in Eq. (3.12) would give rise to an extra factor $1 / 2$ compared to the NLO case. This is compensated however by the factor of 2 in $2 \Re\left[\mathcal{M}_{0} \mathcal{M}_{1}^{*}\right]$.
} 
The second term in the rhs of Eq. (3.14) is the additional divergent contribution to the production cross section caused by a nontrivial analytic continuation of soft loop integrals upon crossing. It reads

$$
\begin{aligned}
\Delta\left[\mathrm{d} \sigma_{\mathrm{RV}}^{\mathrm{div}}\right]= & {\left[\frac{\alpha_{s}(\mu)}{2 \pi}\right]^{2} \frac{2 C_{A} C_{F} \pi^{2}}{\epsilon}\left[\frac{1}{2 v} \ln \left(\frac{1-v}{1+v}\right)+1\right] } \\
& \times\left(\frac{1-v}{v}\right) \mathrm{d} \sigma_{\mathrm{LO}} .
\end{aligned}
$$

Thanks to the argument presented in Sec. II B, we conclude that the cross section for $q \bar{q} \rightarrow V+X$ with massive quarks in the initial state contains noncanceling infrared divergence given by $\Delta\left[\mathrm{d} \sigma_{\mathrm{RV}}^{\mathrm{div}}\right]$. Therefore,

$$
\begin{aligned}
\mathrm{d} \sigma_{\mathrm{NNLO}}= & \Delta\left[\mathrm{d} \sigma_{\mathrm{RV}}^{\mathrm{div}}\right]+\cdots \\
= & {\left[\frac{\alpha_{s}(\mu)}{2 \pi}\right]^{2} \frac{2 C_{A} C_{F} \pi^{2}}{\epsilon}\left[\frac{1}{2 v} \ln \left(\frac{1-v}{1+v}\right)+1\right] } \\
& \times\left(\frac{1-v}{v}\right) \mathrm{d} \sigma_{\mathrm{LO}}+\cdots,
\end{aligned}
$$

where the ellipses stand for finite contributions to the NNLO cross section. Equation (3.16) describes the violation of Bloch-Nordsieck cancellations [29] in the case when two massive quarks collide. It coincides with the expression derived in Refs. [19-21].

We now comment on the result Eq. (3.16). First, we note that in the massless case $v \rightarrow 1$ and the divergence disappears. A simple generalization of this result to the collision of two quarks with unequal masses shows that Eq. (3.16) remains valid provided that $v=$ $\sqrt{1-m_{1}^{2} m_{2}^{2} /\left(p_{1} p_{2}\right)^{2}}$. It follows that the divergence in Eq. (3.16) disappears if only one quark in the initial state is massive.

Moreover, Eq. (3.15) implies that the noncanceling infrared divergences in cross sections with massive quarks in the initial state are power suppressed,

$$
\Delta\left[\mathrm{d} \sigma_{\mathrm{RV}}^{\mathrm{div}}\right] \sim \mathcal{O}\left(\frac{m_{q}^{4}}{m_{V}^{4}}\right) \mathrm{d} \sigma_{\mathrm{LO}}
$$

This behavior is compatible with classic arguments about factorization; see, e.g., Ref. [30] for a review. In fact, a small mass of the quark in the initial state probes the sensitivity of the partonic cross section to long-distance physics. The result Eq. (3.17) then informs us that at the level of logarithmic sensitivity to long-distance effects, the partonic cross section is certainly infrared finite. The noncancellation of infrared divergences at the level of power corrections, as indicated in Eq. (3.17), simply shows that an understanding of factorization for higher twist or, in general, power corrections is required to make calculations with initial-state massive partons self-consistent.

\section{CONCLUSION}

It is well known [19-21] that partonic cross sections computed with massive quarks in the initial state are not infrared finite starting from next-to-next-to-leading order in QCD perturbation theory. We rederived this result in a manner that we find simple and transparent.

The gist of our approach is the relation between infrareddivergent contributions to the manifestly finite decay process $V \rightarrow q \bar{q}+X$ and the production process $q \bar{q} \rightarrow$ $V+X$ that can be studied using analytic continuation. We have explicitly shown that while for the massless case this analytic continuation is harmless through NNLO, the situation is different in a massive theory. There the phase from the analytic continuation of the one-loop soft current combines with a nontrivial imaginary part in the one-loop amplitude and gives rise to an observable effect in the cross section. Our derivation provides a concrete and simple example of problems that one encounters when an analog of a quantum mechanical Coulomb phase manifests itself in massive non-Abelian gauge theories. In fact, it is relatively easy to show that the offending phase is related to a particular double-particle massive cut that encapsulates the long-distance interaction between two incoming massive partons; see, e.g., [24].

Before concluding, we briefly discuss the phenomenological implications of the above results. One may argue that in collider phenomenology one does encounter processes involving massive initial state quarks, e.g., $b \bar{b} \rightarrow H$ and similar. In fact, impressive machinery has been developed for dealing with such processes [31,32]. However, in such cases, one always starts with initial state gluons that subsequently split into a heavy $b \bar{b}$ pair. It is important that massive quarks that originate in such a splitting and participate in the hard scattering process after that are always off-shell. Hence, the average off-shellness of initial state quarks that originate from the gluon splitting $g \rightarrow q \bar{q}$ provides a natural infrared cutoff for processes initiated by massive quarks. For this reason, the infrared divergence shown in Eq. (3.16) can never appear in a realistic setup.

Nevertheless, computations with massive quarks in the initial state can, perhaps, be used to test the sensitivity of partonic cross sections to infrared energy scales that the quark masses may represent. For example, one may wonder to what extent the masses of the colliding quarks affect the transverse momentum distributions of $Z$ and $W$ bosons at low $p_{\perp}$-a question, that may be quite relevant for the determination of the $W$ boson mass at the LHC. Our discussion suggests that, since one starts being sensitive to the off-shellness of quarks only at $\mathcal{O}\left(m_{q}^{4} / m_{V}^{4}\right)$, it should be possible to develop a framework where one keeps track of terms of order $p_{\perp} / m_{q} \sim 1^{10}$ but neglects contributions of

\footnotetext{
${ }^{10}$ For work in this direction, see, e.g., Refs. [33].
} 
order $m_{q}^{4} / m_{V}^{4}$ and beyond. We leave this investigation, as well as the study of its potential phenomenological applications, for the future.

\section{ACKNOWLEDGMENTS}

D. N. would like to thank S. Catani for useful discussions on the original argument. We are grateful to S. Forte, F. Krauss, S. Marzani, and G. Salam for many interesting conversations. We would also like to thank Z. Kunszt and $S$. Marzani for a critical reading of the article. The research of F. C. was partially supported by the ERC Starting Grant 804394 HIPQCD. The research of K. M. was partially supported by the Deutsche Forschungsgemeinschaft (DFG, German Research Foundation) under Grant No. 396021762-TRR 257. The work of D. N. was supported by the ERC Starting Grant REINVENT-
714788. The work of L. T. was supported by the Royal Society through Grant No. URF/R1/191125.

\section{APPENDIX: ANALYTIC CONTINUATION OF THE ONE-LOOP INTEGRALS}

In this appendix, we explicitly compute the analytic continuation of the three integrals $M_{i}$ given in Eq. (3.6) under the $p_{1,2} \rightarrow-p_{1,2}$ transformation. We start with decay kinematics, cf. Fig. 1 . Since $s_{i j}=2 p_{i} \cdot p_{j}$, we find that under the $p_{1,2} \rightarrow-p_{1,2}$ transformation, $s_{1 g} \rightarrow-s_{1 g}$, $s_{2 g} \rightarrow-s_{2 g}$, and $s_{12} \rightarrow s_{12}$. Therefore, to understand how the integrals change under analytic continuation, we only need to study their dependence on $s_{1 g}, s_{2 g}$.

This is most easily achieved if we employ the FeynmanSchwinger parametrization for the integrals $M_{1, \ldots, 3}$. To derive a suitable representation, we start with the identity

$$
\begin{aligned}
\frac{1}{A_{1} A_{2} \ldots A_{n}} & =\Gamma(n) \prod_{i=1}^{n} \int_{0}^{\infty} d x_{i} \frac{\delta\left(1-\sum_{j=1}^{n} x_{j}\right)}{\left[\sum_{i=1}^{n} A_{i} x_{i}\right]^{n}} \\
& =\Gamma(n)\left(\prod_{i \notin \Sigma}^{n} \int_{0}^{\infty} d x_{i}\right)\left(\prod_{i \in \Sigma} \int_{0}^{1} d x_{i}\right) \frac{\delta\left(1-\sum_{j \in \Sigma} x_{j}\right)}{\left[\sum_{i=1}^{n} A_{i} x_{i}\right]^{n}},
\end{aligned}
$$

where $\Sigma$ represents an arbitrary subset of $\{1,2, \ldots, n\}$ [34]. For each integrals $M_{i}$, we choose the set $\Sigma_{i}$ such that it contains the Feynman parameter that is employed for the propagator $1 /\left(-2 p_{2} \cdot k+i \delta\right)$, i.e., $\Sigma=\{3\}$ for $M_{1}$ and $M_{2}$ and $\Sigma=\{4\}$ for $M_{3}$. We find

$$
\begin{aligned}
& M_{1}\left(\epsilon, p_{g} ; p_{1}, p_{2}\right)=-G_{1}(\epsilon) \prod_{i=1}^{2} \int_{0}^{\infty} d x_{i} \frac{\left(x_{1}+x_{2}\right)^{-1+2 \epsilon}}{\left[m^{2}-s_{2 g} x_{2}-i \delta\right]^{1+\epsilon}}, \\
& M_{2}\left(\epsilon, p_{g} ; p_{1}, p_{2}\right)=-G_{2}(\epsilon) \prod_{i=1}^{2} \int_{0}^{\infty} d x_{i} \frac{x_{1}^{-1+2 \epsilon}}{\left[m^{2}\left(1+x_{2}^{2}\right)-s_{1 g} x_{1} x_{2}-s_{12} x_{2}-i \delta\right]^{1+\epsilon}} \\
& M_{3}\left(\epsilon, p_{g} ; p_{1}, p_{2}\right)=-G_{3}(\epsilon) \prod_{i=1}^{3} \int_{0}^{\infty} d x_{i} \frac{\left(x_{1}+x_{2}\right)^{2 \epsilon}}{\left[m^{2}\left(1+x_{3}^{2}\right)-s_{1 g} x_{1} x_{3}-s_{12} x_{3}-s_{2 g} x_{2}-i \delta\right]^{2+\epsilon}},
\end{aligned}
$$

where the explicit form of the $G_{i}(\epsilon)$ is irrelevant in what follows.

It is straightforward to study the dependence of the integrals on $s_{1 g}$ and $s_{2 g}$ using Eq. (A2). We begin with $M_{1}$. By rescaling $x_{i} \rightarrow x_{i} /\left(-s_{2 g}-i \delta\right)$ for $i=1,2$, we find

$$
M_{1}\left(\epsilon, p_{g} ; p_{1}, p_{2}\right)=-G_{1}(\epsilon)\left(-s_{2 g}-i \delta\right)^{-1-2 \epsilon} \prod_{i=1}^{2} \int_{0}^{\infty} d x_{i} \frac{\left(x_{1}+x_{2}\right)^{-1+2 \epsilon}}{\left(m^{2}+x_{2}\right)^{1+\epsilon}},
$$

so that the entire dependence on $s_{2 g}$ factorizes

$$
M_{1}\left(\epsilon, p_{g} ; p_{1}, p_{2}\right) \propto\left(-s_{2 g}-i \delta\right)^{-1-2 \epsilon}=-\left|s_{2 g}\right|^{-1-2 \epsilon} e^{2 i \pi \epsilon} .
$$

This implies

$$
M_{1}\left(\epsilon, p_{g} ;-p_{1},-p_{2}\right) \propto\left|s_{2 g}\right|^{-1-2 \epsilon}
$$


and therefore

$$
M_{1}\left(\epsilon, p_{g} ;-p_{1},-p_{2}\right)=-M_{1}\left(\epsilon, p_{g} ; p_{1}, p_{2}\right) e^{-2 i \pi \epsilon} .
$$

Furthermore, we note that Eq. (A3) implies that in the soft limit

$$
M_{1}\left(\epsilon, p_{g} ; p_{1}, p_{2}\right) \sim E_{g}^{-2 \epsilon} .
$$

We analyze the integral $M_{2}$ in a similar way. In this case, it is sufficient to rescale $x_{1} \rightarrow x_{1} /\left(-s_{1 g}-i \delta\right)$ to find

$$
\begin{aligned}
M_{2}\left(\epsilon, p_{g} ; p_{1}, p_{2}\right)= & G_{2}(\epsilon)\left(-s_{1 g}-i \delta\right)^{-2 \epsilon} \prod_{i=1}^{2} \int_{0}^{\infty} d x_{i} \\
& \times \frac{x_{1}^{-1+2 \epsilon}}{\left[m^{2}\left(1+x_{2}^{2}\right)+x_{1} x_{2}-s_{12} x_{2}-i \delta\right]^{1+\epsilon}} .
\end{aligned}
$$

Hence, the dependence of $M_{2}$ on $p_{1}$ is governed by the following factor:

$$
M_{2}\left(\epsilon, p_{g} ; p_{1}, p_{2}\right) \propto\left(-s_{1 g}-i \delta\right)^{-2 \epsilon} .
$$

Finally, we discuss $M_{3}$. In this case, we rescale $x_{i} \rightarrow x_{i} /\left(-s_{i g}-i \delta\right)$, where we stress that the rescaling is different for the two variables. We obtain

$$
\begin{aligned}
M_{3}\left(\epsilon, p_{g} ; p_{1}, p_{2}\right) & \\
= & -G_{3}(\epsilon) \prod_{i=1}^{3} \int_{0}^{\infty} d x_{i} \\
& \times \frac{\left(\frac{x_{1}}{-s_{1 g}-i \delta}+\frac{x_{2}}{-s_{2 g}-i \delta}\right)^{2 \epsilon}}{\left[m^{2}\left(1+x_{3}^{2}\right)+x_{2}-s_{12} x_{3}+x_{2} x_{3}-i \delta\right]^{2+\epsilon}} .
\end{aligned}
$$

Similarly to what was discussed for $M_{1}$, Eqs. (A9) and (A10) imply

$M_{2,3}\left(\epsilon, p_{g} ;-p_{1},-p_{2}\right)=M_{2,3}\left(\epsilon, p_{g} ; p_{1}, p_{2}\right) e^{-2 i \pi \epsilon}$

and

$$
M_{2,3}\left(\epsilon, p_{g} ; p_{1}, p_{2}\right) \sim E_{g}^{-2 \epsilon} .
$$

Finally, we note that Eq. (3.5) along with Eqs (3.7), (A6), (A7), (A11), and (A12) implies that

$g_{12}^{(1)}\left(\epsilon, p_{g} ;-p_{1},-p_{2}\right)=e^{-2 i \epsilon \pi} g_{12}^{(1)}\left(\epsilon, p_{g} ; p_{1}, p_{2}\right)$

and

$$
g_{12}^{(1)}\left(\epsilon, p_{g} ; p_{1}, p_{2}\right)=\frac{\alpha_{s}}{2 \pi} E_{g}^{-2 \epsilon} \sum_{k=-2}^{\infty}\left[\mathfrak{r}_{k}+i \cdot \dot{\mathfrak{i}}_{k}\right] \epsilon^{k},
$$

with $\mathfrak{r}_{k}$ and $\mathfrak{i}_{k}$ analytic in $E_{g}$. These formulas are used in the main body of the paper to explain the appearance of noncanceling infrared divergencies in collisions of two massive quarks.
[1] D. R. Yennie, S. C. Frautschi, and H. Suura, Ann. Phys. (N.Y.) 13, 379 (1961).

[2] T. Kinoshita, J. Math. Phys. (N.Y.) 3, 650 (1962); T. D. Lee and M. Nauenberg, Phys. Rev. B 133, 1549 (1964).

[3] H. Georgi and H.D. Politzer, Phys. Rev. D 9, 416 (1974).

[4] D. J. Gross and F. Wilczek, Phys. Rev. D 8, 3633 (1973).

[5] R. P. Feynman, Phys. Rev. Lett. 23, 1415 (1969).

[6] R. K. Ellis, H. Georgi, M. Machacek, H. D. Politzer, and G. G. Ross, Phys. Lett. 78B, 281 (1978); R. K. Ellis, H. Georgi, M. Machacek, H. D. Politzer, and G. G. Ross, Nucl. Phys. B152, 285 (1979).

[7] G. Altarelli and G. Parisi, Nucl. Phys. B126, 298 (1977); Y. L. Dokshitzer, Sov. Phys. JETP 46, 641 (1977), http://www.jetp.ac.ru/cgi-bin/dn/e_046_04_0641.pdf; V. N. Gribov and L. N. Lipatov, Sov. J. Nucl. Phys. 15, 438 (1972).

[8] S. B. Libby and G. F. Sterman, Phys. Rev. D 18, 3252 (1978); 18, 4737 (1978).

[9] J. C. Collins, D. E. Soper, and G. F. Sterman, Phys. Lett. 109B, 388 (1982); 126B, 275 (1983); 134B, 263 (1984); Nucl. Phys. B261, 104 (1985); Adv. Ser. Dir. High Energy Phys. 5, 1 (1989).

[10] G. T. Bodwin, Phys. Rev. D 31, 2616 (1985); 34, 3932(E) (1986).

[11] J. C. Collins and D. E. Soper, Annu. Rev. Nucl. Part. Sci. 37, 383 (1987).

[12] J. C. Collins, D. E. Soper, and G. F. Sterman, Nucl. Phys. B308, 833 (1988). 
[13] J. Collins, Foundations of Perturbative QCD (Cambridge University Press, Cambridge, England, 2013), Vol. 32, ISBN 9780521855334.

[14] J. R. Forshaw, A. Kyrieleis, and M. H. Seymour, J. High Energy Phys. 08 (2006) 059; 09 (2008) 128(E); J. Keates and M. H. Seymour, J. High Energy Phys. 04 (2009) 040; M. H. Seymour, arXiv:0710.2733.

[15] J. R. Forshaw, M. H. Seymour, and A. Siodmok, J. High Energy Phys. 11 (2012) 066.

[16] S. Catani, D. de Florian, and G. Rodrigo, J. High Energy Phys. 07 (2012) 026.

[17] L. J. Dixon, E. Herrmann, K. Yan, and H. X. Zhu, J. High Energy Phys. 05 (2020) 135.

[18] C. Anastasiou, C. Duhr, F. Dulat, F. Herzog, and B. Mistlberger, Phys. Rev. Lett. 114, 212001 (2015); C. Duhr, F. Dulat, and B. Mistlberger, Phys. Rev. Lett. 125, 051804 (2020); 125, 172001(E) (2020).

[19] R. Doria, J. Frenkel, and J. C. Taylor, Nucl. Phys. B168, 93 (1980).

[20] C. Di'Lieto, S. Gendron, I. G. Halliday, and C. T. Sachrajda, Nucl. Phys. B183, 223 (1981); J. Frenkel, J. G. M. Gatheral, and J. C. Taylor, Nucl. Phys. B233, 307 (1984); S. Catani, M. Ciafaloni, and G. Marchesini, Nucl. Phys. B264, 588 (1986); S. Catani, Z. Phys. C 37, 357 (1988).

[21] A. Andrasi, M. Day, R. Doria, J. Frenkel, and J. C. Taylor, Nucl. Phys. B182, 104 (1981); C. A. Nelson, Nucl. Phys. B186, 187 (1981); I. Ito, Prog. Theor. Phys. 65, 1466 (1981); N. Yoshida, Prog. Theor. Phys. 66, 269 (1981); 66, 1803 (1981); T. Muta and C. A. Nelson, Phys. Rev. D 25, 2222 (1982); B. F. L. Ward, Phys. Rev. D 78, 056001 (2008).

[22] M. Czakon, Phys. Lett. B 693, 259 (2010); M. Czakon, Nucl. Phys. B849, 250 (2011).

[23] F. Caola, K. Melnikov, and R. Röntsch, Eur. Phys. J. C 77, 248 (2017).

[24] S. Catani, S. Dittmaier, and Z. Trocsanyi, Phys. Lett. B 500, 149 (2001).
[25] S. Catani and M.H. Seymour, Nucl. Phys. B485, 291 (1997); B510, 503(E) (1998).

[26] S. Catani and M. Grazzini, Nucl. Phys. B570, 287 (2000).

[27] S. Catani and M. Grazzini, Nucl. Phys. B591, 435 (2000).

[28] I. Bierenbaum, M. Czakon, and A. Mitov, Nucl. Phys. B856, 228 (2012).

[29] F. Bloch and A. Nordsieck, Phys. Rev. 52, 54 (1937).

[30] R. K. Ellis, W. J. Stirling, and B. R. Webber, $Q C D$ and Collider Physics (Cambridge University Press, Cambridge, England, 2011), Vol. 8, ISBN 9780521545891.

[31] M. A. G. Aivazis, J. C. Collins, F. I. Olness, and W. K. Tung, Phys. Rev. D 50, 3102 (1994); M. Cacciari, M. Greco, and P. Nason, J. High Energy Phys. 05 (1998) 007; S. Forte, E. Laenen, P. Nason, and J. Rojo, Nucl. Phys. B834, 116 (2010); S. Forte, D. Napoletano, and M. Ubiali, Phys. Lett. B 751, 331 (2015); M. Bonvini, A. S. Papanastasiou, and F. J. Tackmann, J. High Energy Phys. 11 (2015) 196; S. Forte, D. Napoletano, and M. Ubiali, Phys. Lett. B 763, 190 (2016); M. Bonvini, A. S. Papanastasiou, and F. J. Tackmann, J. High Energy Phys. 10 (2016) 053.

[32] F. Maltoni, G. Ridolfi, and M. Ubiali, J. High Energy Phys. 07 (2012) 022; 04 (2013) 095(E); M. Lim, F. Maltoni, G. Ridolfi, and M. Ubiali, J. High Energy Phys. 09 (2016) 132; F. Krauss and D. Napoletano, Phys. Rev. D 98, 096002 (2018); S. Forte, T. Giani, and D. Napoletano, Eur. Phys. J. C 79, 609 (2019).

[33] P. Pietrulewicz, D. Samitz, A. Spiering, and F. J. Tackmann, J. High Energy Phys. 08 (2017) 114; P. M. Nadolsky, N. Kidonakis, F. I. Olness, and C. P. Yuan, Phys. Rev. D 67, 074015 (2003); S. Berge, P. M. Nadolsky, and F. I. Olness, Phys. Rev. D 73, 013002 (2006); A. Belyaev, P. M. Nadolsky, and C.P. Yuan, J. High Energy Phys. 04 (2006) 004.

[34] H. Cheng and T. T. Wu, in Expanding Protons: Scattering at High-Energies (MIT-PR, Cambridge, MA, 1987), p. 285. 